\title{
Simulations of Disk Galaxy Formation in their Cosmological Context
}

\author{
Simon D.M. White \\ Max Planck Institute for Astrophysics \\ Karls-Schwarzschild-Strasse 1 \\ D-85741 Garching, Germany \\ E-mail: swhite@mpa-Garching.mpg.de
}

\begin{abstract}
Together with the discovery of the accelerated expansion of the present Universe and measurements of large-scale structure at low redshift, observations of the cosmic microwave background have established a standard paradigm in which all cosmic structure grew from small fluctuations generated at very early times in a flat universe which today consists of $72 \%$ dark energy, $23.5 \%$ dark matter and $4.5 \%$ ordinary baryons. The CMB sky provides us with a direct image of this universe when it was 400,000 years old and very nearly uniform. The galaxy formation problem is then to understand how observed galaxies with all their regularity and diversity arose from these very simple initial conditions. Although gravity is the prime driver, many physical processes appear to play an important role in this transformation, and direct numerical simulation has become the principal tool for detailed investigation of the complex and strongly nonlinear interactions between them.

The evolution of structure in the gravitationally dominant Cold Dark Matter distribution can now be simulated in great detail, provided the effects of the baryons are ignored, and there is general consensus for the results on scales relevant to the formation of galaxies like our own. The basic nonlinear units are so-called "dark matter halos", slowly rotating, triaxial, quasiequilibrium systems with a universal cusped density profile and substantial substructure in the form of a host of much less massive subhalos which are concentrated primarily in their outer regions.
\end{abstract}

Attempts to include the baryons, and so to model the formation of the visible parts of galaxies, have given much more diverse results. It has been known for 30 years that substantial feedback, presumably from stellar winds and supernovae, is required to prevent overcooling of gas and excessive star formation in the early stages of galaxy assembly. When realistic galaxy formation simulations first became possible in the early 1990's, this problem was immediately confirmed. Without effective feedback, typical halos produced galaxies which were too massive, too concentrated and had too little disk to be consistent with observation.

Simple models for disk formation from the mid 1990's show that the angular momentum predicted for collapsing dark halos is sufficient for them to build a disk population similar to that observed. Direct simulations have repeatedly failed to confirm this picture, however, because nonlinear effects lead to substantial transfer of angular momentum between the various components. In most cases the condensing baryonic material loses angular momentum to the dark matter, and the final galaxy ends up with a disk that is too compact or contains too small a fraction of the stars.

These problems have been reduced as successive generations of simulations have dramatically improved the numerical resolution and have introduced "better" implementations of feedback (i.e. more successful at building disks). Despite this, no high-resolution simulation has so far been able to produce a present-day disk galaxy with a bulge-to-disk mass ratio much less than one in a proper $\Lambda$ CDM context. Such galaxies are common in the real Universe; our own Milky Way is a good example. The variety of results obtained by different groups show that this issue is very sensitive to how star formation and feedback are treated, and all implementations of these processes to date have been much too schematic to be confident of their predictions. 
The major outstanding issues I see related to disk galaxies and their formation are the following: Do real disk galaxies have the NFW halos predicted by the $\Lambda$ CDM cosmology? If not, could the deviations have been produced by the formation of the observed baryonic components, or must the basic structure formation picture be changed? How are Sc and later type galaxies made? Why don't our simulations produce them? What determines which galaxies become barred and which not? Can we demonstrate that secular evolution produces the observed population of (pseudo)bulges from pre-existing disks? How does the observed population of thin disks survive bombardment by substructure and the other transient potential fluctuations expected in $\Lambda \mathrm{CDM}$ halos? Is a better treatment of feedback really the answer? If so, can we demonstrate it using chemical abundances as fossil tracers? And how can we best use observations at high redshift to clarify these formation issues?

Keywords. cosmology: theory, dark matter - galaxies: formation - galaxies: structure - galaxies: evolution - galaxies: halos

The presentation made at the meeting is available at:

http://www.mpa-garching.mpg.de/ swhite/IAU254.pdf

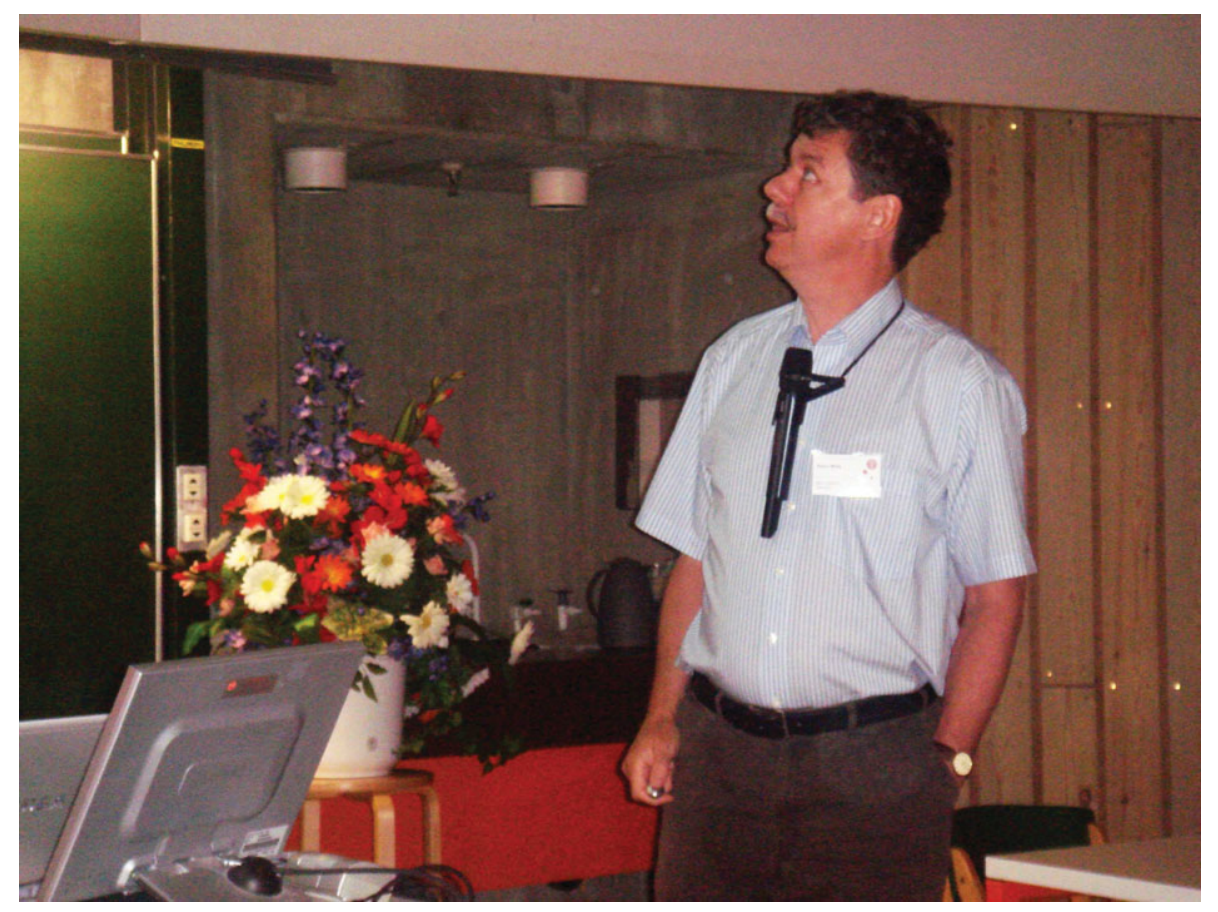

Simon White setting the scene for the symposium. 